\title{
Performance studies of RPC detectors operated with new environmentally friendly gas mixtures in presence of LHC-like radiation background
}

\author{
Beatrice Mandelli, ${ }^{a, *}$ Roberto Guida $^{a}$ and Gianluca Rigoletti ${ }^{b}$ \\ ${ }^{a}$ CERN, \\ Espl. des Particules 1, Meyrin, Switzerland \\ ${ }^{b}$ University Claude Bernard Lyon I, \\ Lyon, France \\ E-mail: beatrice.mandelli@cern.ch
}

Resistive Plate Chamber (RPC) detectors are widely used thanks to their excellent time resolution and low production cost. At the CERN LHC experiments, the large RPC systems are operated in avalanche mode thanks to a Freon-based gas mixture containing $\mathrm{C}_{2} \mathrm{H}_{2} \mathrm{~F}_{4}$ and $\mathrm{SF}_{6}$, both greenhouse gases with a very high global warming potential (GWP). The search of new environmentally friendly gas mixtures is advisable for reducing greenhouse gas emissions, costs as well as to optimize RPC performance and possible detector aging issues.

Several hydrofluorocarbons, hydrofluoroolefins (HFOs) and innovative industrial alternative to $\mathrm{SF}_{6}$ gases with very low GWP have been identified as possible replacements of $\mathrm{C}_{2} \mathrm{H}_{2} \mathrm{~F}_{4}$ and $\mathrm{SF}_{6}$. More than 60 environmentally friendly gas mixtures have been investigated on $2 \mathrm{~mm}$ single-gap RPCs. The RPC detectors have been tested in laboratory conditions and at the CERN Gamma Irradiation Facility (GIF++), which provides a high energy muon beam combined with an intense gamma source allowing to simulate the background expected at HL-LHC.

RPCs performance have been studied at different gamma rates with the new environmentally friendly gases by measuring efficiency, streamer probability, rate capability, induced charge, cluster size and time resolution. Encouraging results of RPC operation in avalanche mode have been obtained with 4 and 5 components gas mixtures. To finalize the studies, the RPCs are now operated under gas recirculation with the selected new gas mixture and exposed to the intense gamma radiation at the CERN GIF++ facility for evaluating possible long-term aging effects, gas damage due to radiation and compatibility of LHC gas system with new gases.

40th International Conference on High Energy physics - ICHEP2020

July 28 - August 6, 2020

Prague, Czech Republic (virtual meeting)

\footnotetext{
${ }^{*}$ Speaker
} 


\section{Introduction}

The Resistive Plate Chamber (RPC) detectors [1] are employed at CERN in the ATLAS, CMS and ALICE experiments for the muon trigger systems. They are operated with an humidified gas mixture (40\% relative humidity) made of $\mathrm{C}_{2} \mathrm{H}_{2} \mathrm{~F}_{4}, \mathrm{SF}_{6}$ and $\mathrm{iC}_{4} \mathrm{H}_{10}$ that allows operation in avalanche mode at high rate $\left(\sim 1 \mathrm{kHz} / \mathrm{cm}^{2}\right) . \mathrm{C}_{2} \mathrm{H}_{2} \mathrm{~F}_{4}$ and $\mathrm{SF}_{6}$ are greenhouse gases (GHGs) with a global warming potential (GWP) of 1430 and 22800 respectively. Given the large detector volume and the use of expensive GHGs, the RPC gas systems are operated with gas recirculation systems.

The European Union set a F-gas regulation [2] starting from January 2015 with the aim of reducing the usage of F-gases in Europe. It is therefore foreseeable that F-gases availability would be uncertain in Europe for the future and their price could raise possibly making gas detectors operation very costly. The search of new environmentally friendly gas mixtures for RPCs is therefore advisable since these detectors will be operated for the next decades in the LHC experiments.

\section{Alternatives to $\mathrm{C}_{2} \mathrm{H}_{2} \mathrm{~F}_{4}$ and $\mathrm{SF}_{6}$ for $\mathrm{RPC}$ detectors}

The alternatives to GHGs are nowadays dictated by the industrial market, which is evolving to meet the challenges presented by the EU regulations. The $\mathrm{C}_{2} \mathrm{H}_{2} \mathrm{~F}_{4}$, also called R134a, is employed as refrigerant in industry. In the last 10 years, research and development activities were focused on HydroFluoroOlefins (HFOs) as potential new refrigerants, having a very low GWP (less than 6). With respect to R134a, HFOs contain one carbon more and a double-bond. The HFO isomer selected for the characterisation of RPC is HFO1234ze as it is not classified as flammable.

In the recent years, research has also focused in alternatives to $\mathrm{SF}_{6}$, which is mainly used as insulator for high-voltage plants. The $3 \mathrm{M}$ Company has developed two new sustainable alternatives to $\mathrm{SF}_{6}$ with a high dielectric strength and low GWP: $3 \mathrm{M}$ Novec $5110\left(\mathrm{CF}_{3} \mathrm{C}(\mathrm{O}) \mathrm{CF}\left(\mathrm{CF}_{3}\right)_{2}\right)$ and $3 \mathrm{M}$ Novec $4710\left(\left(\mathrm{CF}_{3}\right)_{2} \mathrm{CFCN}\right)$. Two other gases have been studied in this work as alternatives to $\mathrm{SF}_{6}$ : the $\mathrm{CF}_{3} \mathrm{I}$, which has a GWP of 0.4 and is very electronegative but it is toxic, and the $\mathrm{C}_{4} \mathrm{~F}_{8} \mathrm{O}$, which has a high GWP ( 8700) but has good electronegative properties.

\section{Characterisation of RPC detector with eco-friendly gases}

In this work the research is focused in finding possible new eco-friendly gas mixtures able to reproduce the RPC performance observed with the current $\mathrm{C}_{2} \mathrm{H}_{2} \mathrm{~F}_{4}$-based gas mixture used at the LHC experiments (called standard RPC gas mixture in the following). In this case it is not possible to replace most of the infrastructure (i.e. high voltage systems, cables, front-end electronics, etc.) as well as the detectors themselves. Therefore the replacement of the gas mixture is particularly challenging as it is not possible to adapt any infrastructure or the detector to obtain good results with the eco-friendly gas mixtures.

\subsection{Experimental set-up}

A dedicated set-up has been built for the characterization of RPC detectors with different gas mixtures. Two High Pressure Laminate RPCs with a $2 \mathrm{~mm}$ gas gap, a surface of about $80 \times 100$ $\mathrm{cm}^{2}$ and read-out strips $2.1 \mathrm{~cm}$ wide are used.The induced signals on the strips are directly acquired 
with a CAEN Digitizer V1730 without amplification. For each waveform the signals are processed to obtain pulse height, charge and timing distributions.

For each gas mixture, the RPC performance is evaluated by measuring efficiency, streamer probability, rate capability, induced charge, cluster size and time resolution. A detailed description of the set-up and data analysis procedure can be found in [3].

\subsection{RPC performance with HFO-based gas mixtures}

The direct replacement of $\mathrm{C}_{2} \mathrm{H}_{2} \mathrm{~F}_{4}$ with HFO in the standard gas mixture is not suitable for the operation of LHC RPC detectors: an applied voltage of more than $13 \mathrm{kV}$ is necessary to start the ionisation process (higher with respect to the HV that can be used).

To overcome this problem $\mathrm{He}$ and $\mathrm{CO}_{2}$ were added to the HFO-based gas mixture to lower the HV working point [3]. An addition of about $30-40 \%$ of $\mathrm{He}$ is enough to reduce the HV working point. Nevertheless, the streamer probability increases drastically, probably due to the presence of only HFO. The reintroduction of a small quantity of $\mathrm{C}_{2} \mathrm{H}_{2} \mathrm{~F}_{4}$ in the mixture reduces the signal charge. The mixture HFO/ $\mathrm{C}_{2} \mathrm{H}_{2} \mathrm{~F}_{4} / \mathrm{He} / \mathrm{iC}_{4} \mathrm{H}_{10} / \mathrm{SF}_{6}(37.45 / 37.45 / 20 / 4.5 / 0.6)$ allows to obtain RPC foremost parameters very similar to the standard gas mixture (Figure 1 left). Also the use of $\mathrm{CO}_{2}$ is interesting: $10 \% \mathrm{CO}_{2}$ reduces the working point by $800 \mathrm{~V}$. An addition of $40-50 \% \mathrm{of}_{2}$ is necessary to reach the desired working point. Also in this case a fraction of $\mathrm{C}_{2} \mathrm{H}_{2} \mathrm{~F}_{4}$ is kept in the new gas mixture and the $\mathrm{SF}_{6}$ concentration is increased to $1 \%$ to suppress streamers (Figure 1 right).
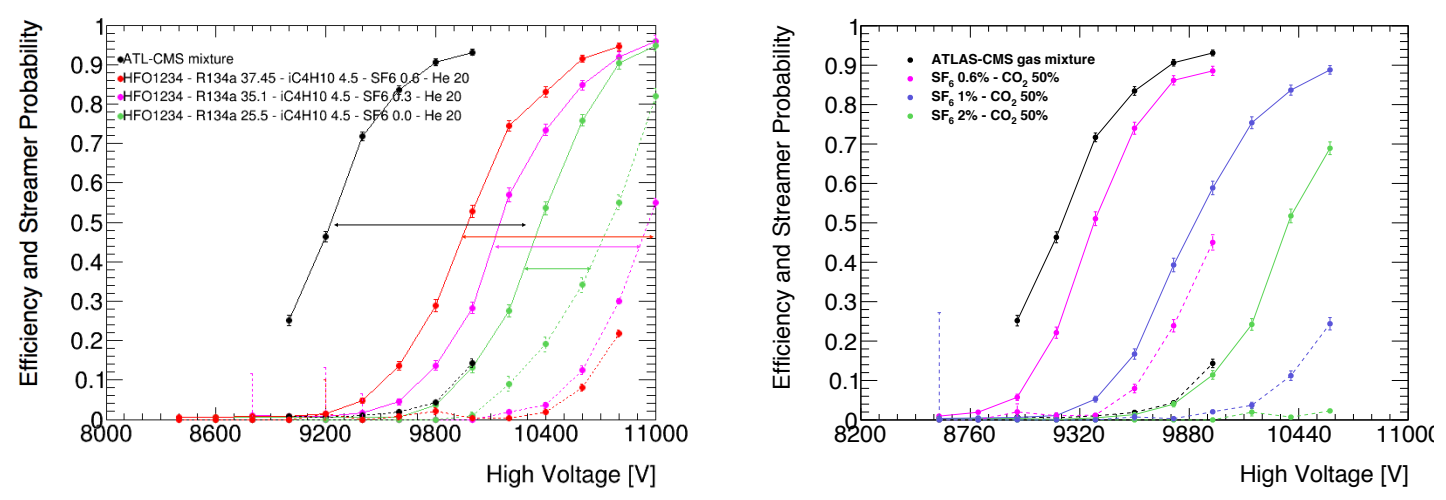

Figure 1: Efficiency (continuous line) and streamer probability (dotted lines) as a function of the $\mathrm{HV}$ for gas mixtures with different concentrations of $\mathrm{SF}_{6}, 4.5 \% \mathrm{iC}_{4} \mathrm{H}_{10}, 20 \% \mathrm{He}$ (left plot) or $50 \% \mathrm{CO}_{2}$ (right plot) and the remaining gas is $\mathrm{C}_{2} \mathrm{H}_{2} \mathrm{~F}_{4}$ and $\mathrm{HFO}$ in the same proportions.

\subsection{RPC performance with alternatives to $\mathrm{SF}_{6}$}

The studies on alternatives to $\mathrm{SF}_{6}$ have been performed by using the standard gas mixture as reference and replacing the $\mathrm{SF}_{6}$ with the new candidates. For each gas, different concentrations were tested to better characterise its properties. The best results for each gas mixture are summarised in Figure 2. Despite the high dielectric strength, the Novec 5110 does not allow RPC operation at a low streamer probability even with a concentration of $2 \%$. An hypothesis is that Novec 5110 breaks inside the gap being sensitive to UV radiation. On the contrary only $0.1 \%$ of Novec 4710 is enough 
to reach the same streamer probability of the standard gas mixture. Nevertheless Novec 4710 may react with water and it can be problematic since the RPC gas mixture has $40 \%$ relative humidity. Outside the Novec family, the $\mathrm{C}_{4} \mathrm{~F}_{8} \mathrm{O}$ and $\mathrm{CF}_{3} \mathrm{I}$ have been tested: good results have been obtained but both gases do not satisfy all necessary requirements.

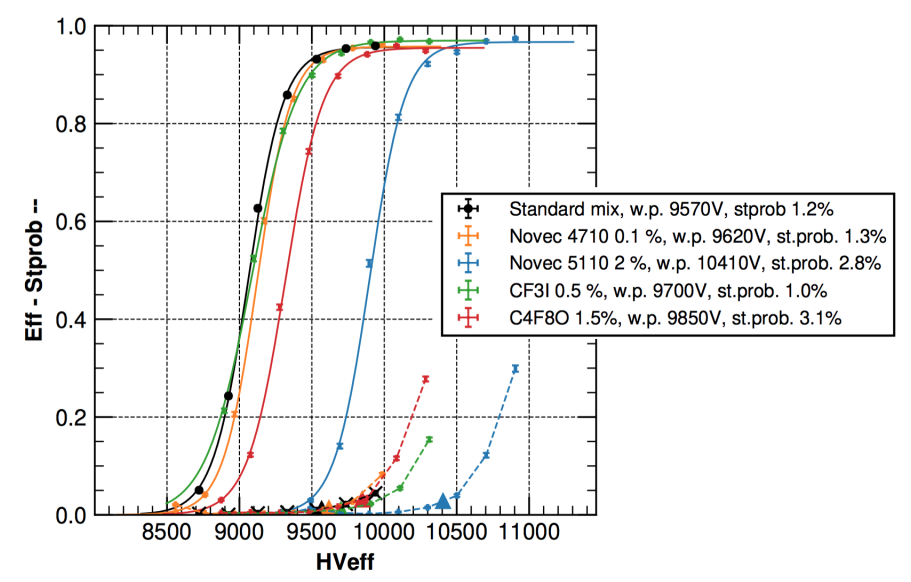

Figure 2: Efficiency (continuos line) and streamer probability (dotted line) for the standard gas mixture and the gas mixture containing $\mathrm{SF}_{6}$ alternatives wih the best results.

\section{RPC operated with eco-friendly gas mixtures in presence of gamma background radiation}

The performance of RPC with eco-friendly gas mixtures has to be studied also in LHC-like conditions, i.e. in presence of high background radiation and under gas recirculation. This can be achieved at the CERN Gamma Irradiation Facility ( $\mathrm{GIF}++$ ), which provides radiation from a ${ }^{137} \mathrm{Cs}$ source of $14 \mathrm{TBq}$ combined with a high energy charged particle beam [4].

RPC performance have been compared between the standard gas mixture and two mixtures based on the addition of $\mathrm{HFO}$ and $\mathrm{CO}_{2}: \mathrm{C}_{2} \mathrm{H}_{2} \mathrm{~F}_{4} / \mathrm{HFO} / \mathrm{CO}_{2} / \mathrm{iC}_{4} \mathrm{H}_{10} / \mathrm{SF}_{6}(27.25 / 27.25 / 40 / 4.5 / 1)$ and $\mathrm{C}_{2} \mathrm{H}_{2} \mathrm{~F}_{4} / \mathrm{HFO} / \mathrm{CO}_{2} / \mathrm{iC}_{4} \mathrm{H}_{10} / \mathrm{SF}_{6}(22.25 / 22.25 / 50 / 4.5 / 1)$. Different absorption factors (ABSs) have been used to have a gamma rate spacing from $0.7 \mathrm{kHz} / \mathrm{cm}^{2}$ (ABS 22000) to $55.3 \mathrm{kHz} / \mathrm{cm}^{2}$ (ABS 100), corresponding to about the maximum gamma rate that the RPCs will see in CMS during the HL-LHC phase [5].

The test-beam measurements confirmed the laboratory tests: the HV shift of HFO-based gas mixtures is the same in presence of high background radiation (Figure 3 left) and the streamer probability is about a factor two higher for HFO gas mixtures. The detector currents of HFO gas mixtures are about $40 \%$ higher with respect to standard gas mixture. The efficiency and streamer probability have been studied at different ABS: the curves are completely overlapped demonstrating that the muon detection efficiency is not affected by the background rate (up to $55.3 \mathrm{kHz} / \mathrm{cm}^{2}$ ). A meticulous study has been performed on the pulse charges for avalanche and streamer at different background rates (Figure 3 right). The avalanche charge is higher for these $\mathrm{CO}_{2}$-based gas mixtures with respect to the standard gas mixture while the streamer charge is very similar for the three gas mixtures. 

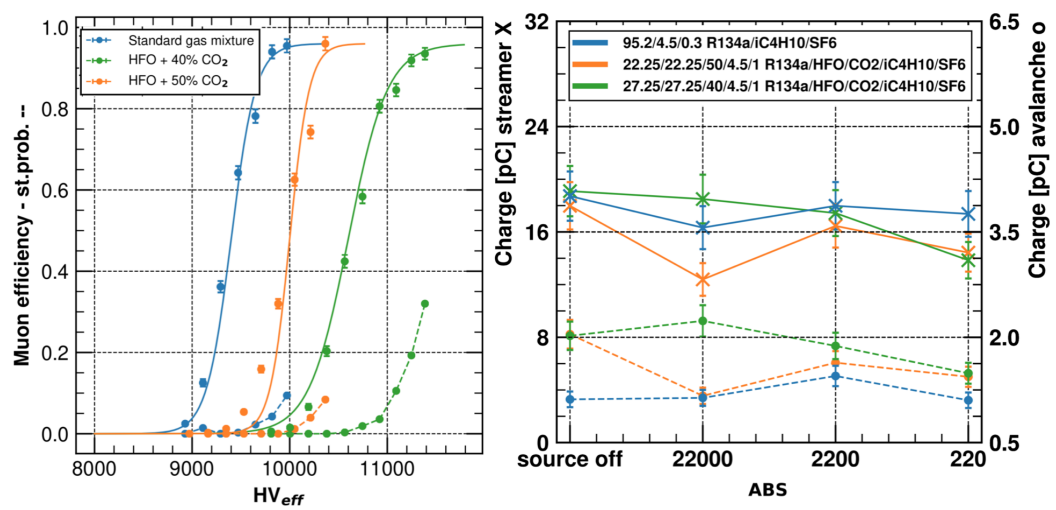

Figure 3: On the left: efficiency and streamer probability of RPCs operated at $40 \mathrm{kHz} / \mathrm{cm}^{2}$ for the three gas mixtures tested. On the right: avalanche and streamer (dotted and continuous line) charges at different ABSs.

\subsection{Creation of impurities with HFO-based gas mixtures}

Under the effects of radiation and electric field, the $\mathrm{C}_{2} \mathrm{H}_{2} \mathrm{~F}_{4}$ molecule breaks into several compounds and free fluoride ions [6], which can accumulate under gas recirculation [7] and could be harmful for the long-term detector operation. Furthermore in presence of water, the free fluoride ions become hydrofluoric acid (HF), which is a very reactive compound. The presence of these impurities was observed in past tests and recently in LHC RPC systems in LHC Run 2 [8].

Systematic studies were performed at GIF++ to understand if the HFO goes under this breaking process. $\mathrm{F}^{-}$measurements were performed at different voltages and ABSs for the standard gas mixture and the gas mixture $\mathrm{C}_{2} \mathrm{H}_{2} \mathrm{~F}_{4} / \mathrm{HFO} / \mathrm{CO}_{2} / \mathrm{iC}_{4} \mathrm{H}_{10} / \mathrm{SF}_{6}(27.25 / 27.25 / 40 / 4.5 / 1)$. Figure 4 shows the $\mathrm{F}^{-}$production of the two gas mixtures at different rates: the $\mathrm{F}^{-}$production is four times higher in the HFO gas mixture. By considering the concentration of the components in the two gas mixtures, it can be deduced that the $\mathrm{C}_{3} \mathrm{H}_{2} \mathrm{~F}_{4}$ breaks about 10 times more than the $\mathrm{C}_{2} \mathrm{H}_{2} \mathrm{~F}_{4}$. The test highlights
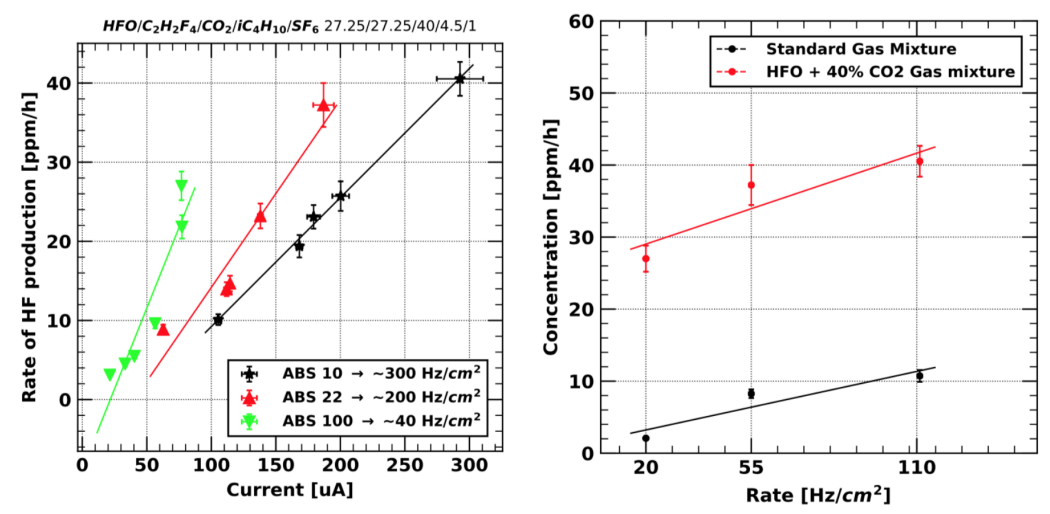

Figure 4: On the left: rate of HF production as a function of RPC current for different ABSs for the selected $\mathrm{HFO}$ gas mixture. On the right: rate of $\mathrm{HF}$ production as a function of rate for the standard gas mixture and $\mathrm{C}_{2} \mathrm{H}_{2} \mathrm{~F}_{4} / \mathrm{HFO} / \mathrm{CO}_{2} / \mathrm{iC}_{4} \mathrm{H}_{10} / \mathrm{SF}_{6}(27.25 / 27.25 / 40 / 4.5 / 1)$.

that if HFOs will be used in LHC experiments, the formation of impurities will be higher with respect to $\mathrm{C}_{2} \mathrm{H}_{2} \mathrm{~F}_{4}$ and their effect on long-term detector operation has to be studied in details. 


\section{Conclusions}

This work is focused in the search of an environmentally friendly gas mixture that is compatible with the current ATLAS and CMS RPC systems. The direct replacement of $\mathrm{C}_{2} \mathrm{H}_{2} \mathrm{~F}_{4}$ and $\mathrm{SF}_{6}$ with the corresponding eco-friendly alternatives available in industry is not suitable to achieve good detector performance. In particular, the use of $\mathrm{HFO}$ as replacement of $\mathrm{C}_{2} \mathrm{H}_{2} \mathrm{~F}_{4}$ entails the addition of an inert gas $\left(\mathrm{He}\right.$ or $\left.\mathrm{CO}_{2}\right)$ to lower the $\mathrm{HV}$ working point. Only with few 5-components gas mixtures it was possible to obtain RPC performance rather similar to the one of the standard gas mixture. Satisfying results were obtained with some alternatives of $\mathrm{SF}_{6}$ but studies are still needed to verify their impact on long-term operation.

RPC performance were studies with muon beam at the GIF++ using two eco-friendly gas mixtures based on $\mathrm{HFO}$ and $\mathrm{CO}_{2}$ : currents, streamer probability and avalanche charge are slightly higher with respect to RPC standard gas mixture. Furthermore studies on the production of impurities revealed that HFO breaks more easily than $\mathrm{C}_{2} \mathrm{H}_{2} \mathrm{~F}_{4}$ creating several impurities and HF. Based on these results, RPC performance with HFO-based gas mixture have to be studied in details and a long term test of RPC operation at GIF++ has to be envisaged.

\section{References}

[1] R. Santonico and R. Cardarelli, Developments of Resistive Plate Counters, Nucl. Instrum. Meth. A 187 (1981) 377.

[2] Regulation (EU) No 517/2014 of the European Parliament and of the Council on Fluorinated Greenhouse Gases and Repealing Regulation (EC) No 842/2006.

[3] M. Capeans, R. Guida, B. Mandelli, Characterization of RPC operation with new environmental friendly mixtures for LHC application and beyond, 2016 JINST 11 C07016, doi:10.1088/1748-0221/11/07/C07016.

[4] R. Guida, GIF++: A new CERN Irradiation Facility to test large-area particle detectors for the High-Luminosity LHC program, PoS (ICHEP2016) 260.

[5] CMS Collaboration, The Phase-2 Upgrade of the CMS Muon Detectors, CERN-LHCC-2017012 - CMS-TDR-016.

[6] R. Guida et al., Results about HF production and bakelite analysis for the CMS Resistive Plate Chambers, Nucl. Instrum. Meth. A, A 594 (2008) S140-S147, doi.org/10.1016/j.nima.2008.06.009.

[7] M. Capeans, I. Glushkov, R. Guida, F. Hahn, S. Haider, Optimization of a closed-loop gas system for the operation of Resistive Plate Chambers at the Large Hadron Collider experiments, Nucl. Instrum. Meth. A, A 661 (2012) S214-S221, doi:10.1016/j.nima.2010.08.077.

[8] B. Mandelli, R. Guida, M. Gagliardi, M. Marchisone, Gas mixture quality monitoring for the RPC detectors at the LHC experiments, JINST 14 C09006, https://doi.org/10.1088/17480221/14/09/C09006. 